\title{
Leader-Following Consensus of Multiagent Systems with Time-Varying Delays via Impulsive Control
}

\author{
Zhiyong Ye, ${ }^{1}$ Yungen Chen, ${ }^{1}$ and Hua Zhang ${ }^{1,2}$ \\ ${ }^{1}$ Institute of Mathematics and Statistics, Chongqing University of Technology, Chongqing 400054, China \\ ${ }^{2}$ Department of Mathematics and Computer Science, Tongren College, Tongren 554300, China \\ Correspondence should be addressed to Zhiyong Ye; yezhiyong1966@163.com
}

Received 11 November 2013; Accepted 14 January 2014; Published 10 March 2014

Academic Editor: Marcelo Moreira Cavalcanti

Copyright (c) 2014 Zhiyong Ye et al. This is an open access article distributed under the Creative Commons Attribution License, which permits unrestricted use, distribution, and reproduction in any medium, provided the original work is properly cited.

\begin{abstract}
The leader-following consensus problem for delayed multiagent systems is investigated over stochastic switching topologies via impulsive control method. A distributed consensus protocol is proposed based on sample data information. The convergence analysis for such algorithm over undirected and directed networks is provided, and some sufficient conditions to guarantee the consensus are also established. It is shown that delayed networks can achieve consensus even information is exchanged among followers just at some discrete moments. At last, some numerical examples are given to illustrate the effectiveness of the proposed protocols.
\end{abstract}

\section{Introduction}

Recently, there has been an increasing attention in the consensus problems of multiagent systems because of their wide applications in many fields such as flocking $[1,2]$, mobile sensor networks [3], cooperative control [4], and formation control [5]. Both discrete-time consensus model $[1,6-8]$ and continuous-time consensus model [9-14] are studied. For example, Vicsek et al. first proposed a discretetime model for multiagent systems [6]. Then, Jadbabaie et al. [7] and Savkin [8] successfully gave explanations of Vicsek's model, respectively. Based on algebraic graph theory, Olfati-Saber and Murray showed that the average-consensus problems can be solved if the directed network topology is strongly connected and balanced [9]. Further, Ren and Beard extended [9] and proved that consensus can be achieved if and only if the network topology has a spanning tree [10].

As we know, in the real world, especially in neural processing and signal transmission, time delay has to be taken into consideration [15]. Because time delay may cause unexpected dynamical behaviors such as oscillation and instability [11]. In [9], Olfati-Saber and Murray considered consensus with fixed communication delay and derived the bound of delay as well as a necessary and sufficient condition to guarantee the consensus. In [12, 13], Lee and Spong and
$\mathrm{Lu}$ et al. considered consensus problem with nonuniform time-delays, respectively, and proved that the first-order multiagent system can realize consensus asymptotically for any fixed and finite time delays. In [14], Liu et al. defined a new concept of consensus in order to investigate consensus of multiagent systems with unbounded time-varying delays.

It should be noticed that in practical life, some biological and physical systems are characterized by abrupt changes of states at some time instants [16]. These phenomena are usually regarded as impulsive effects of communication links among agents [17]. Impulsive effects exist due to unreliable communication links [18]. Inspired by the impulsive phenomena, some effective impulsive methods are applied into different models [17, 19-24]. In [19], impulsive effect is studied for fuzzy delayed systems through the Takagi-Sugeno model and some criteria for stability are derived by virtue of the Razumikhin technique. Li et al. investigated synchronization of coupled delayed neural networks via impulsive control [20]. Recently, Zhang and Jiang addressed synchronization problem for nonlinear discrete chaotic systems with impulsive effects [21]. Zhou et al. studied impulsive synchronization of coupled harmonic oscillators over fixed and switching topology, respectively [17]. Wu et al. proposed an average consensus protocol to study a delayed multiagent system with control inputs missing [22]. Guan et al. investigated 
impulsive consensus seeking in directed network topology [23]. It should be noticed that impulsive control has some advantages compared with continuous control. For example, it is flexible, energy-saving, and cost-saving. What is more, it has been proved that the impulsive control approach is effective and robust [21].

To the best of our knowledge, the leader-following consensus problem is becoming more and more popular recently [25-32]. The leader's motion is independent, whereas the followers' motion is influenced by the leader and its neighbors. Therefore, in order to stabilize a multiagent system, we can control the leader (some leaders) instead of all the agents in a system. This helps to save energy and reduce control cost [25]. Moreover, it is shown that the leaderfollowing configuration can also enhance the communication and orientation of the flock [26]. In [27], Hong et al. gave a scheme to track a leader by studying a consensus problem over undirected networks with switching topology. $\mathrm{Hu}$ and Hong derived some conditions to guarantee leader-following consensus for delayed multiagent system over fixed and switching topologies, respectively [28]. Song et al. proposed a pinning control protocol for nonlinear multiagent systems with double integrator and addressed what kind of agents and how many agents should be pinned [29]. Recently, quasiconsensus of second-order multiagent systems with a leader was studied by Wang and Cao [30]. A finite-time consensus protocol for multiagent networks was discussed by Yang et al. from a new perspective [31]. $M$-matrix strategies are designed by Song et al. to pin a second-order nonlinear multiagent system [32].

Motivated by the aforementioned works, we study leaderfollowing consensus of delayed multiagent systems via impulsive control and propose a protocol based on sampled data information. Compared with pure discrete-time or pure continuous-time model, this problem is more complicated. The analysis becomes difficult if the time delay is timevarying and the topology is switching. We analyse this problem by virtue of algebraic graph theory, the Lyapunov control approach, and generalized Halanay inequality. The contribution of this paper is threefold. First, we propose a more practical protocol by considering communication delay, impulsive effect, and leader-following configuration. Second, without assuming that the interaction graph is strongly connected (connected) or balanced, or has a directed spanning tree, we study this problem over general network topologies. Third, we generalize the works of $[9,10]$ and show advantages such as time-saving and cost-saving via simulation compared with the works of $[9,10,22,23]$.

The paper is organized as follows. Section 2 presents some preliminaries. The leader-following consensus protocol via impulsive control is analyzed in Section 3. Some simulation results are provided in Section 4. Finally, conclusions are given in Section 5.

\section{Preliminaries}

2.1. Notations and Definition. Throughout this paper, some mathematical notations and definitions are used here. Let $\mathbb{R}$ and $\mathbb{N}$ represent real numbers and positive natural numbers, respectively. $\mathbb{R}^{N}$ and $\mathbb{R}^{N \times N}$ stand for real vector space and real matrix space, respectively. Let $I_{N}\left(O_{N}\right)$ be identity (zero) matrix and $1_{N}=(1, \ldots, 1)^{\top} \in \mathbb{R}^{N}$. Let $A^{\top}$ and $A^{w}=(A+$ $\left.A^{\top}\right) / 2$ stand for the transpose and the symmetric part of $A \in$ $\mathbb{R}^{N \times N} \cdot \lambda_{\max }(A)$ denotes the maximum eigenvalues of matrix $A$ with the spectra norm $\|A\|=\left[\lambda_{\max }\left(A^{\top} \cdot A\right)\right]^{1 / 2}$. The symbol $\otimes$ denotes the Kronecker product.

Let the function $\psi:[-\tau, 0] \rightarrow \mathbb{R}^{N}$ form the linear space $P C\left([-\tau, 0], \mathbb{R}^{N}\right)$ with the norm $\|\cdot\|_{\tau}=\sup _{-\tau \leq u \leq 0}\|\psi(u)\|$. The function $\psi$ has the following property: $\psi(t)$ is continuous everywhere except a finite number of points $\bar{t}$, where $\psi\left(\bar{t}^{+}\right)$ and $\psi(\bar{t})$ exist and $\psi\left(\bar{t}^{+}\right)=\psi(\bar{t})$. Let $\psi\left(\bar{t}^{+}\right)=\lim _{u \rightarrow 0^{+}} \psi(t+$ $u), \psi(\bar{t})=\lim _{u \rightarrow 0^{-}} \psi(t+u)$, and we denote $[\psi(t)]_{\tau}=$ $\sup _{-\tau \leq u \leq 0}\{\psi(t+u)\},[\psi(t)]_{\tau^{-}}=\sup _{-\tau \leq u<0}\{\psi(t+u)\}$ [33]. For a function $g(t) \in C[Q, \mathbb{R}], Q=[0,+\infty), D^{+} g(t) \triangleq$ $\overline{\lim }_{h \rightarrow 0^{+}}(1 / h)(g(t+h)-g(t))$ is called Dini derivative. Matrix dimensions are assumed to be compatible for algebraic operations.

2.2. Graph Theory. We use a graph to describe the information exchange among agents in a multiagent system. Let $G(V, E, A)$ be a weighted directed network of order $N$, with the set of nodes $V=\{1,2, \ldots, N\}$, the set of directed edges $E \subset V \times V$, and a weighted adjacency matrix $A=\left[a_{i j}\right] \in \mathbb{R}^{N \times N}$ with nonnegative adjacency elements $a_{i j}$ [9]. The element of $A$ is defined as $a_{i j}>0$ if $(j, i) \in E$; otherwise, $a_{i j}=0$ for all $i \neq j$, which means that if node $i$ can receive information from node $j$, then $a_{i j}>0$, and we suppose that $a_{i i}=0$ for all $i \in V$ [10]. If $a_{i j}=a_{j i}>0$; that is, $A$ is a symmetric matrix, we call the corresponding graph of $A$ as undirected graph. Note that the undirected graphs are special cases of directed graph.

A path from node $j$ to $i$ in directed (undirected) graph is a sequence of edges $\left(j, k_{1}\right),\left(k_{1}, k_{2}\right), \ldots,\left(k_{m}, i\right)$ with distinct nodes $k_{l}(l=1, \ldots, m)$ [34]. If there is a path between any pair of distinct nodes of a graph, we call this graph strongly connected. In a directed graph, we say the graph has a directed spanning tree if there exists at least one node called root which has a directed path to all the other nodes [29]. Moreover, if a graph $G$ has such root labeled 0 , we say that the node 0 is globally reachable in $G$. This condition is much weaker than strong connectedness [28]. The neighbor set of node $i$ is defined by $N_{i}=\{j \in V \mid(j, i) \in E\}$. The Laplacian matrix of weighted graph is defined by $L=\left[l_{i j}\right] \in \mathbb{R}^{N \times N}$, where $l_{i i}=\sum_{j=1}^{N} a_{i j}$ and $l_{i j}=-a_{i j}, i \neq j$. In this paper, we suppose that all the interconnection graphs have a globally reachable node 0 .

2.3. Model Description. Many people studied the first-order consensus protocol as follows $[9,10]$ :

$$
\dot{x}_{i}(t)=\sum_{v_{j} \in N_{i}} a_{i j}(t)\left[x_{j}(t-\tau)-x_{i}(t-\tau)\right] .
$$

For example, Olfati-Saber and Murray studied this protocol over undirected networks with constant time delay and fixed topology and suggested that the network topology and the designed protocol are very important to the performance and the communication cost [9]. In this paper, we consider 
a group of $m+1$ agents, including one leader and $m$ identical followers (or agents if no confusion arises) and propose the following impulsive leader-following consensus protocol:

$$
\begin{aligned}
\dot{x}_{i}(t)= & \sum_{v_{j} \in N_{i}} a_{i j}^{(n)}(t)\left[x_{j}(t-\tau(t))-x_{i}(t-\tau(t))\right] \delta\left(t-t_{n}\right) \\
& +\alpha_{i}^{(n-1)}(t)\left(x_{0}(t)-x_{i}(t)\right), \quad n \in \mathbb{N}, i=1,2, \ldots, m,
\end{aligned}
$$

where $n:[0, \infty) \rightarrow \Gamma=\{1, \ldots, \zeta\}$ is a switching signal that determines the network topology at $t=t_{n}$. We define two graphs: a graph $G$ that is formed only by $m$ followers and a graph $\widetilde{G}$ that is formed by a leader and $m$ followers. Let $\bar{G}=$ $\left\{\widetilde{G}_{1}, \ldots, \widetilde{G}_{\zeta}\right\}$ denote the set of all possible interconnection graphs with a common node set $\bar{V}=\{0,1,2, \ldots, m\}$. Then the communication topology is a subset of $\bar{G}$. Denote the set $\widehat{G}=\left\{G_{1}, \ldots, G_{\zeta}\right\}$ with a common node set $\widehat{V}=\{1,2, \ldots, m\}$. Obviously $G_{k}$ is a part of $\widetilde{G}_{k}(k \in \Gamma)$. The time sequence $\left\{t_{n}\right\}$ satisfies $0=t_{0}<t_{1}<\cdots<t_{n}<\cdots, \lim _{n \rightarrow \infty} t_{n}=\infty$. Let $x_{i} \in \mathbb{R}^{N}$ be the value of the $i$ th agent, which may represent physical quantities such as position, temperature, and voltage [9]. $\delta(t)$ is the Dirac impulsive function, which indicates that the states of (2) have jumps at $t_{n}$. We suppose that $\tau(t)$ is a continuously differentiable function satisfying $0<\tau(t) \leq \tau$, $a_{i j}^{(n)}(t)=d c_{i j}^{(n)}(t), \alpha_{i}^{(n-1)}(t)=\alpha b_{i}^{(n-1)}(t)$, where $d$ and $\alpha$ are the weighting factors and $d>0, \alpha>0 . c_{i j}^{(n)}$ is the element of adjacency matrix $A^{(n)}$, where $A^{(n)}$ is the adjacency matrix of communication topology $G^{(n)}$ at time $t_{n} \cdot b_{i}^{(n-1)}(t)=1$ if agent $i$ is connected to the leader at time interval $\left[t_{n-1}, t_{n}\right)$; otherwise, $b_{i}^{(n-1)}(t)=0$.

The dynamics of the leader are described as follows:

$$
\dot{x}_{0}(t)=0 \text {, }
$$

where $x_{0} \in \mathbb{R}^{N}$ is the value of the leader. Obviously, the motion of the leader is independent of followers.

Without loss of generality, we suppose that $N=1$, and thus using the properties of Dirac impulsive function, the system (2) can be rewritten as

$$
\begin{gathered}
\dot{x}(t)=-B^{(n-1)}\left(x(t)-1_{m} \otimes x_{0}(t)\right), \\
t \in\left[t_{n-1}, t_{n}\right), \quad t \geq 0, \quad n \in \mathbb{N}, \\
\Delta x\left(t_{n}\right)=x\left(t_{n}\right)-x\left(t_{n}^{-}\right)=-L^{(n)} x\left(t_{n}-\tau\left(t_{n}\right)\right), \quad t=t_{n},
\end{gathered}
$$

where $B^{(n-1)}=\operatorname{diag}\left(\alpha_{1}^{(n-1)}, \ldots, \alpha_{m}^{(n-1)}\right), L^{(n)}=\left[l_{i j}^{(n)}\right]_{m \times m}$ represents the Laplacian matrix of graph $G^{(n)}$, and is defined by

$$
l_{i j}^{(n)}=\left\{\begin{array}{cc}
\sum_{r=1, r \neq i}^{m} a_{i r}^{(n)}, & j=i, \\
-a_{i j}^{(n)}(t), & j \neq i .
\end{array}\right.
$$

Let $e(t)=x(t)-1_{m} \otimes x_{0}(t)$; thus, we can rewrite system (4) as

$$
\begin{gathered}
\dot{e}(t)=-B^{(n-1)} e(t), \quad t \in\left[t_{n-1}, t_{n}\right), t \geq 0, n \in \mathbb{N}, \\
\Delta e=\left(t_{n}\right)-L^{(n)} e\left(t_{n}-\tau\left(t_{n}\right)\right), \quad t=t_{n} .
\end{gathered}
$$

Remark 1. Recently, many works studied consensus problem, and most of the network topologies are assumed to be connected (strong connected) or have a spanning tree [9, $10,22,23]$. In this paper, we focus on the leader-following consensus of delayed multiagent systems with impulsive effects over a general network topology. For example, for a group of migratory birds, neither all the birds can receive information from the leader, nor can the birds communicate information all the time [35]. We suppose that only a few birds can obtain the leader's information. Each bird only communicates with its neighbors at a series of discrete time with time delay and updates its states in a manner of impulse jumps. So system (4) can be regarded as hybrid system.

\section{Convergence Analysis}

In this section, we will give convergence analysis of this problem over undirected and directed networks, respectively. From the system (2), we know that information is exchanged among followers only at some discrete moments. So in the time interval $\left[t_{n}-\tau\left(t_{n}\right), t_{n}\right]$, we suppose that the frequency of information exchange among followers is $\xi_{n}$, and the states of the system (2) have jumps at $t_{n-\xi_{n}+1}, t_{n-\xi_{n}+2}, \ldots, t_{n}$.

\subsection{Undirected Networks over Switching Topology}

Theorem 2. Suppose that every communication topology in $\bar{G}$ has a globally reachable node 0 . If there exists a constant $\lambda>0$, such that for all $n \in \mathbb{N}$, the following condition is satisfied:

$$
0<p_{n-1}+q_{n-1} e^{2 \lambda \tau} \leq 1,
$$

where

$$
\begin{gathered}
p_{n}=\max _{b \leq i \leq m} \frac{1+\xi_{n} e^{\alpha^{\prime} \tau} \lambda_{i}^{(n)} \max _{n-\xi_{n}+1 \leq l \leq n} \lambda_{i}^{(l)}}{\left(1+\lambda_{b}\left(L^{(n)}\right)\right)^{2}} \\
q_{n}=\max _{b \leq i \leq m} \frac{\xi_{n} e^{\alpha^{\prime} \tau} \lambda_{i}^{(n)} \max _{n-\xi_{n}+1 \leq l \leq n} \lambda_{i}^{(l)}+\xi_{n}^{2} e^{2 \alpha^{\prime} \tau} \lambda_{i}^{(n) 2} \max _{n-\xi_{n}+1 \leq l \leq n} \lambda_{i}^{(l) 2}}{\left(1+\lambda_{b}\left(L^{(n)}\right)\right)^{2}} .
\end{gathered}
$$

And $\lambda_{b}(\cdot)$ represents the minimal positive eigenvalue of $L^{(n)}$. $p_{0}=1, q_{0}=0, \alpha^{\prime}=\max _{i \in m}\left(\max _{n-\xi_{n} \leq j \leq n-1} \alpha_{i}\right)$, and $e$ and $\dot{\tau}$ are the natural constant and the maximum of the time delays, respectively. $\lambda_{i}^{(n)}$ and $\alpha_{i}^{(n)}(i=b, b+1, \ldots, m)$ represent the ith eigenvalues of $L^{(n)}$ and $B^{(n)}$, respectively. Then the system (2) achieves consensus globally exponentially.

Proof. Since the Laplacian matrix $L^{(n)}$ of undirected networks is positive semidefinite [9], there exists a real orthogonal matrix $H^{(n)}$, so that $C^{(n)}=H^{(n) \top} L^{(n)} H^{(n)}$, where $C^{(n)}=$ $\operatorname{diag}\left(\lambda_{1}^{(n)}, \ldots, \lambda_{m}^{(n)}\right)$, and $\lambda_{i}^{(n)}(i=1,2, \ldots, m)$ represents the 
$i$ th eigenvalues of $L^{(n)}$. Let $\left[\theta_{1}, \ldots, \theta_{m}\right]^{\top}=H^{(n) \top}\left[e_{1}, \ldots, e_{m}\right]^{\top}$, then

$$
\begin{gathered}
\dot{\theta}(t)=-B^{(n-1)} \theta(t), \quad t \in\left[t_{n-1}, t_{n}\right), t \geq 0, n \in \mathbb{N}, \\
\Delta \theta\left(t_{n}\right)=H^{(n) \top} \Delta e\left(t_{n}\right)=-C^{(n)} \theta\left(t_{n}-\tau\left(t_{n}\right)\right), \quad t=t_{n} .
\end{gathered}
$$

That is,

$$
\begin{gathered}
\dot{\theta}_{i}(t)=-\alpha_{i}^{(n-1)} \theta_{i}(t), \quad t \in\left[t_{n-1}, t_{n}\right), t \geq 0, n \in \mathbb{N}, \\
\Delta \theta_{i}\left(t_{n}\right)=-\lambda_{i}^{(n)} \theta_{i}\left(t_{n}-\tau\left(t_{n}\right)\right), \quad t=t_{n}, \quad i=1,2, \ldots, m,
\end{gathered}
$$

where $\lambda_{i}^{(n)}$ and $\alpha_{i}^{(n)}(i=1,2, \ldots, m)$ represent the $i$ th eigenvalues of $L^{(n)}$ and $B^{(n)}$, respectively.

Construct a Lyapunov function $V(t)=\theta^{\top}(t) \theta(t)=$ $\sum_{i=1}^{m} \theta_{i}^{2}(t)$, if $t \in\left[t_{n-1}, t_{n}\right), n \in \mathbb{N}$; then

$$
D^{+} V(t)=2 \sum_{i=1}^{m} \theta_{i}(t) \dot{\theta}_{i}(t)=-2 \alpha_{i}^{(n-1)} V(t) \leq 0 .
$$

Let $t_{n-\xi_{n}+1}-t_{n-\xi_{n}}=\Delta_{n-\xi_{n}}, t_{n-\xi_{n}+2}-t_{n-\xi_{n}+1}=\Delta_{n-\xi_{n}+1}, \ldots, t_{n}-$ $t_{n-1}=\Delta_{n-1}, t_{n}-\tau\left(t_{n}\right)-t_{n-\xi_{n}}=\Delta^{\prime}$. From (10), we have

$$
\begin{gathered}
\theta_{i}\left(t_{n-\xi_{n}+1}^{-}\right)=\theta_{i}\left(t_{n-\xi_{n}}\right) e^{-\alpha_{i}^{\left(n-\xi_{n}\right)} \Delta_{n-\xi_{n}}} \\
\theta_{i}\left(t_{n-\xi_{n}+1}\right)-\theta_{i}\left(t_{n-\xi_{n}+1}^{-}\right) \\
=-\lambda_{i}^{\left(n-\xi_{n}+1\right)} \theta_{i}\left(t_{n-\xi_{n}+1}-\tau\left(t_{n-\xi_{n}+1}\right)\right) .
\end{gathered}
$$

Then, we have

$$
\begin{aligned}
& \theta_{i}\left(t_{n-\xi_{n}}\right) \\
& =e^{\alpha_{i}^{\left(n-\xi_{n}\right)} \Delta_{n-\xi_{n}}} \theta_{i}\left(t_{n-\xi_{n}+1}^{-}\right) \\
& =e^{\alpha_{i}^{\left(n-\xi_{n}\right)} \Delta_{n-\xi_{n}}} \\
& \times\left[\theta_{i}\left(t_{n-\xi_{n}+1}\right)+\lambda_{i}^{\left(n-\xi_{n}+1\right)} \theta_{i}\left(t_{n-\xi_{n}+1}-\tau\left(t_{n-\xi_{n}+1}\right)\right)\right] \\
& =e^{\alpha_{i}^{\left(n-\xi_{n}\right)} \Delta_{n-\xi_{n}}} \\
& \times\left[e^{\alpha_{i}^{\left(n-\xi_{n}+1\right)} \Delta_{n-\xi_{n}+1}} \theta_{i}\left(t_{n-\xi_{n}+2}\right)\right. \\
& \left.+e^{\alpha_{i}^{\left(n-\xi_{n}+1\right)} \Delta_{n-\xi_{n}+1}} \lambda_{i}^{\left(n-\xi_{n}+2\right)} \theta_{i}\left(t_{n-\xi_{n}+2}-\tau\left(t_{n-\xi_{n}+2}\right)\right)\right] \\
& +e^{\alpha_{i}^{\left(n-\xi_{n}\right)} \Delta_{n-\xi_{n}}}\left[\lambda_{i}^{\left(n-\xi_{n}+1\right)} \theta_{i}\left(t_{n-\xi_{n}+1}-\tau\left(t_{n-\xi_{n}+1}\right)\right)\right] \\
& =e^{\sum_{j=n-\xi_{n}}^{n-1} \alpha_{i}^{(j)} \Delta_{j}} \theta_{i}\left(t_{n}\right) \\
& +\sum_{s=1}^{\xi_{n}} e^{\sum_{k=0}^{s-1} \alpha_{i}^{\left(n-\xi_{n}+k\right)} \Delta_{n-\xi_{n}+k}} \lambda_{i}^{\left(n-\xi_{n}+s\right)} \theta_{i} \\
& \times\left(t_{n-\xi_{n}+s}-\tau\left(t_{n-\xi_{n}+s}\right)\right) .
\end{aligned}
$$

So we can obtain

$$
\begin{aligned}
\Delta \theta_{i}\left(t_{n}\right)= & -\lambda_{i}^{(n)} \theta_{i}\left(t_{n}-\tau\left(t_{n}\right)\right) \\
= & -\lambda_{i}^{(n)} \theta_{i}\left(t_{n-\xi_{n}}\right) e^{-\alpha_{i}^{\left(n-\xi_{n}\right)}\left(t_{n}-\tau\left(t_{n}\right)-t_{n-\xi_{n}}\right)} \\
= & -\lambda_{i}^{(n)} e^{\left(\sum_{j=n-\xi_{n}}^{n-1} \alpha_{i}^{(j)} \Delta_{j}-\alpha_{i}^{\left(n-\xi_{n}\right)} \Delta^{\prime}\right)} \theta_{i}\left(t_{n}\right) \\
& -\left\{\sum_{s=1}^{\xi_{n}} \lambda_{i}^{(n)} e^{\left(\sum_{k=0}^{s-1} \alpha_{i}^{\left(n-\xi_{n}+k\right)} \Delta_{n-\xi_{n}+k}-\alpha_{i}^{\left(n-\xi_{n}\right)} \Delta^{\prime}\right)}\right. \\
& \left.\times \lambda_{i}^{\left(n-\xi_{n}+s\right)} \theta_{i}\left(t_{n-\xi_{n}+s}-\tau\left(t_{n-\xi_{n}+s}\right)\right)\right\} .
\end{aligned}
$$

Then, we can get

$$
\begin{aligned}
&\left(1+\lambda_{i}^{(n)} e^{\left(\sum_{j=n-\xi_{n}}^{n-1} \alpha_{i}^{(j)} \Delta_{j}-\alpha_{i}^{\left(n-\xi_{n}\right)} \Delta^{\prime}\right)}\right) \theta_{i}\left(t_{n}\right) \\
&=\theta_{i}\left(t_{n}^{-}\right)-\left\{\sum_{s=1}^{\xi_{n}} \lambda_{i}^{(n)} e^{\left(\sum_{k=0}^{s-1} \alpha_{i}^{\left(n-\xi_{n}+k\right)} \Delta_{n-\xi_{n}+k}-\alpha_{i}^{\left(n-\xi_{n}\right)} \Delta^{\prime}\right)}\right. \\
&\left.\quad \times \lambda_{i}^{\left(n-\xi_{n}+s\right)} \theta_{i}\left(t_{n-\xi_{n}+s}-\tau\left(t_{n-\xi_{n}+s}\right)\right)\right\} .
\end{aligned}
$$

Since $L^{(n)}$ is positive semidefined, let the minimal positive eigenvalue of $L^{(n)}$ be $\lambda_{b}\left(L^{(n)}\right)$; then the eigenvalues of $L^{(n)}$ can be ordered as $0=\lambda_{1}\left(L^{(n)}\right)=\cdots=\lambda_{b-1}\left(L^{(n)}\right)<\lambda_{b}\left(L^{(n)}\right) \leq$ $\cdots \leq \lambda_{m}\left(L^{(n)}\right) \stackrel{\text { def }}{=} \lambda_{\max }\left(L^{(n)}\right)[36]$.

So for $i=1, \ldots, b-1$, from (10), we can obtain

$$
V\left(t_{n}\right) \leq V\left(t_{n}^{-}\right) .
$$

For $i=b, \ldots, m$, let $\alpha^{\prime}=\max _{i \in m}\left(\max _{n-\xi_{n} \leq j \leq n-1} \alpha_{i}^{(j)}\right)$, and let $\tau$ be the maximum of the time delays; then from (15) and using the inequality $\left(\sum_{i=1}^{m} x_{i}\right)^{2} \leq m \sum_{i=1}^{m} x_{i}^{2}, 2 x_{1} x_{2} \leq x_{1}^{2}+x_{2}^{2}$, we can obtain that

$$
\begin{aligned}
(1 & \left.+\lambda_{b}\left(L^{(n)}\right)\right)^{2} V\left(t_{n}\right) \\
\leq & \sum_{i=1}^{m}\left(1+\lambda_{i}^{(n)} e^{\left(\sum_{j=n-\xi_{n}}^{n-1} \alpha_{i}^{(j)} \Delta_{j}-\alpha_{i}^{\left(n-\xi_{n}\right)} \Delta^{\prime}\right)}\right)^{2} \theta_{i}^{2}\left(t_{n}\right) \\
\leq & \sum_{i=1}^{m} \theta_{i}^{2}\left(t_{n}^{-}\right)+2 e^{\alpha^{\prime} \tau} \lambda_{i}^{(n)} \\
& \times \max _{n-\xi_{n}+1 \leq l \leq n} \lambda_{i}^{(l)} \sum_{i=1}^{m}\left[\sum_{s=1}^{\xi_{n}} \theta_{i}\left(t_{n}^{-}\right) \theta_{i}\left(t_{n-\xi_{n}+s}-\tau\left(t_{n-\xi_{n}+s}\right)\right)\right] \\
& +e^{2 \alpha^{\prime} \tau} \lambda_{i}^{(n) 2} \\
& \times \max _{n-\xi_{n}+1 \leq l \leq n} \lambda_{i}^{(l) 2} \sum_{i=1}^{m}\left[\sum_{s=1}^{\xi_{n}} \theta_{i}\left(t_{n-\xi_{n}+s}-\tau\left(t_{n-\xi_{n}+s}\right)\right)\right]^{2} \\
\leq & V\left(t_{n}^{-}\right)+e^{\alpha^{\prime} \tau} \lambda_{i}^{(n)}
\end{aligned}
$$




$$
\begin{aligned}
& \times \max _{n-\xi_{n}+1 \leq l \leq n} \lambda_{i}^{(l)} \sum_{i=1}^{m}\left\{\sum_{s=1}^{\xi_{n}}\left[\theta_{i}^{2}\left(t_{n}^{-}\right)+\theta_{i}^{2}\left(t_{n-\xi_{n}+s}-\tau\left(t_{n-\xi_{n}+1}\right)\right)\right]\right\} \\
& +\xi_{n} e^{2 \alpha^{\prime} \tau} \lambda_{i}^{(n) 2} \\
& \times \max _{n-\xi_{n}+1 \leq l \leq n} \lambda_{i}^{(l) 2} \sum_{i=1}^{m}\left[\sum_{s=1}^{\xi_{n}} \theta_{i}^{2}\left(t_{n-\xi_{n}+s}-\tau\left(t_{n-\xi_{n}+s}\right)\right)\right] \\
& \leq\left(1+\xi_{n} e^{\alpha^{\prime} \tau} \lambda_{i}^{(n)} \max _{n-\xi_{n}+1 \leq l \leq n} \lambda_{i}^{(l)}\right) V\left(t_{n}^{-}\right) \\
& +\left(\xi_{n} e^{\alpha^{\prime} \tau} \lambda_{i}^{(n)} \max _{n-\xi_{n}+1 \leq l \leq n} \lambda_{i}^{(l)}+\xi_{n}^{2} e^{2 \alpha^{\prime} \tau} \lambda_{i}^{(n) 2} \max _{n-\xi_{n}+1 \leq l \leq n} \lambda_{i}^{(l) 2}\right) \\
& \times\left[V\left(t_{n}\right)\right]_{2 \tau^{-}},
\end{aligned}
$$

where $\left[V\left(t_{n}\right)\right]_{2 \tau^{-}}=\sup _{t_{n}-2 \tau \leq u<t_{n}} V(u)$.

Combine with (16) and (17); from the above proof, we can obtain

$$
\begin{gathered}
D^{+} V(t) \leq 0, \quad t \neq t_{n}, \quad t \geq 0, n \in \mathbb{N}, \\
V\left(t_{n}^{+}\right) \leq p_{n} V\left(t_{n}^{-}\right)+q_{n}\left[V\left(t_{n}\right)\right]_{2 \tau^{-}}, \quad t=t_{n},
\end{gathered}
$$

where $p_{n}, q_{n}$ are defined in Theorem 2; then according to Lemma 1 in [33], the system (2) achieves consensus globally exponentially. That completes the proof of Theorem 2 .

\subsection{Directed Networks over Switching Topology}

Theorem 3. Suppose that node 0 is globally reachable in every communication topology of $\bar{G}$. If there exists a constant $\lambda>0$, such that for all $n \in \mathbb{N}$, the following condition is satisfied:

$$
\begin{gathered}
0<p_{n-1}+q_{n-1} e^{2 \lambda \tau} \leq 1, \\
p_{n}=\left(1+\varepsilon \xi_{n}\left\|L^{(n)}\right\|_{n-\xi_{n}+1 \leq s \leq n}\left\|L^{(s)}\right\|\right. \\
\left.+\varepsilon\left(\xi_{n}+1\right) \Delta\left\|L^{(n)}\right\|_{n-\xi_{n} \leq l \leq n-1}\left\|B^{(l)}\right\|\right) \\
\times\left(1+2 \lambda_{c}\left(L^{(n) w}\right)+\lambda_{d}\left(L^{(n) \top} L^{(n)}\right)\right)^{-1}, \\
q_{n}=\left(\varepsilon^{-1} \xi_{n}\left\|L^{(n)}\right\| \max _{n-\xi_{n}+1 \leq s \leq n}\left\|L^{(s)}\right\|\right. \\
\left.+\varepsilon^{-1}\left(\xi_{n}+1\right) \Delta\left\|L^{(n)}\right\| \max _{n-\xi_{n} \leq l \leq n-1}\left\|B^{(l)}\right\|\right) \\
\times\left(1+2 \lambda_{c}\left(L^{(n) w}\right)+\lambda_{d}\left(L^{(n) \top} L^{(n)}\right)\right)^{-1}
\end{gathered}
$$

$$
\begin{aligned}
& +\left(\xi_{n}^{2}\left\|L^{(n)}\right\|_{n-\xi_{n}+1 \leq s \leq n}^{2} \max _{n}\left\|L^{(s)}\right\|^{2}\right. \\
& \left.+\left(\xi_{n}+1\right)^{2} \Delta^{2}\left\|L^{(n)}\right\|_{n-\xi_{n} \leq l \leq n-1}^{2} \max _{n}\left\|B^{(l)}\right\|^{2}\right) \\
& \times\left(1+2 \lambda_{c}\left(L^{(n) w}\right)+\lambda_{d}\left(L^{(n) \top} L^{(n)}\right)\right)^{-1} \\
& +\left(\xi_{n}\left(\xi_{n}+1\right) \Delta\left\|L^{(n)}\right\|^{2}\right. \\
& \left.\quad \times \max _{n-\xi_{n}+1 \leq s \leq n}\left\|L^{(s)}\right\| \max _{n-\xi_{n} \leq l \leq n-1}\left\|B^{(l)}\right\|\right) \\
& \times\left(1+2 \lambda_{c}\left(L^{(n) w}\right)+\lambda_{d}\left(L^{(n) \top} L^{(n)}\right)\right)^{-1},
\end{aligned}
$$

where $\Delta=\sup _{n \in \mathbb{N}}\left\{t_{n+1}-t_{n}\right\}, \varepsilon$ is a constant and $\varepsilon>0, p_{0}=$ $1, q_{0}=0$, and $\lambda_{c}(\cdot)$ and $\lambda_{d}(\cdot)$ represent the minimal positive eigenvalue of $L^{(n) w}$ and $L^{(n) \top} L^{(n)}$, respectively. Then the system (2) achieves consensus globally exponentially.

Proof. Suppose that in the time interval $\left[t_{n}-\tau\left(t_{n}\right), t_{n}\right]$, the frequency of information exchange is $\xi_{n}$; then

$$
\begin{aligned}
e\left(t_{n-\xi_{n}}\right)= & e\left(t_{n-\xi_{n}+1}^{-}\right)+\int_{t_{n-\xi_{n}}}^{t_{n-\xi_{n}+1}^{-}} B^{\left(n-\xi_{n}\right)} e(t) d t \\
= & e\left(t_{n-\xi_{n}+1}\right) \\
& +L^{\left(n-\xi_{n}+1\right)} e\left(t_{n-\xi_{n}+1}-\tau\left(t_{n-\xi_{n}+1}\right)\right) \\
& +\int_{t_{n-\xi_{n}}}^{t_{n-\xi_{n}+1}^{-}} B^{\left(n-\xi_{n}\right)} e(t) d t \\
= & e\left(t_{n}\right)+\sum_{s=n-\xi_{n}+1}^{n} L^{(s)} e\left(t_{s}-\tau\left(t_{s}\right)\right) \\
& +\sum_{j=1}^{\xi_{n}} \int_{t_{n-j}}^{t_{n-j+1}^{-}} B^{(n-j)} e(t) d t .
\end{aligned}
$$

Thus, we have

$$
\begin{aligned}
\Delta e\left(t_{n}\right)= & -L^{(n)} e\left(t_{n}-\tau\left(t_{n}\right)\right) \\
= & -L^{(n)}\left[e\left(t_{n-\xi_{n}}\right)-\int_{t_{n-\xi_{n}}}^{t_{n}-\tau\left(t_{n}\right)} B^{\left(n-\xi_{n}\right)} e(t) d t\right] \\
=-L^{(n)} & {\left[e\left(t_{n}\right)+\sum_{s=n-\xi_{n}+1}^{n} L^{(s)} e\left(t_{s}-\tau\left(t_{s}\right)\right)\right.} \\
& +\sum_{j=1}^{\xi_{n}} \int_{t_{n-j}}^{t_{n-j+1}^{-}} B^{(n-j)} e(t) d t \\
& \left.-\int_{t_{n-\xi_{n}}}^{t_{n}-\tau\left(t_{n}\right)} B^{\left(n-\xi_{n}\right)} e(t) d t\right] .
\end{aligned}
$$


Now we rewrite the system (6) as

$$
\begin{aligned}
\dot{e}(t)=-B^{(n-1)} e(t), \quad & t \in\left[t_{n-1}, t_{n}\right), t \geq 0, n \in \mathbb{N}, \\
\left(I+L^{(n)}\right) e\left(t_{n}\right)=e\left(t_{n}^{-}\right) & \\
& -L^{(n)}\left[\sum_{s=n-\xi_{n}+1}^{n} L^{(s)} e\left(t_{s}-\tau\left(t_{s}\right)\right)\right. \\
& +\sum_{j=1}^{\xi_{n}} \int_{t_{n-j}}^{t_{n-j+1}^{-}} B^{(n-j)} e(t) d t \\
& \left.-\int_{t_{n-\xi_{n}}}^{t_{n}-\tau\left(t_{n}\right)} B^{\left(n-\xi_{n}\right)} e(t) d t\right], \quad t=t_{n} .
\end{aligned}
$$

Define the Lyapunov function as $V(t)=e^{\top}(t) e(t)$, if $t \in$ $\left[t_{n-1}, t_{n}\right), n \in \mathbb{N}$,

$$
D^{+} V(t)=2 e^{\top}(t) \dot{e}(t)=-2 e^{\top}(t) B^{(n-1)} e(t) \leq 0 .
$$

Since $L^{(n) w}$ and $L^{(n) \top} L^{(n)}$ are positive semidefined, denote the minimal positive eigenvalues of $L^{(n) w}$ and $L^{(n) \top} L^{(n)}$ as $\lambda_{c}\left(L^{(n) w}\right)$ and $\lambda_{d}\left(L^{(n) \top} L^{(n)}\right)$, respectively. Then, $0=$ $\lambda_{1}\left(L^{(n) w}\right)=\cdots=\lambda_{c-1}\left(L^{(n) w}\right)<\lambda_{c}\left(L^{(n) w}\right) \leq \cdots \leq$ $\lambda_{m}\left(L^{(n) w}\right) \stackrel{\text { def }}{=} \lambda_{\max }\left(L^{(n) w}\right), 0=\lambda_{1}\left(L^{(n) \top} L^{(n)}\right)=\cdots=$ $\lambda_{d-1}\left(L^{(n) \top} L^{(n)}\right)<\lambda_{d}\left(L^{(n) \top} L^{(n)}\right) \leq \cdots \leq \lambda_{m}\left(L^{(n) \top} L^{(n)}\right) \stackrel{\text { def }}{=}$ $\lambda_{\text {max }}\left(L^{(n) \top} L^{(n)}\right)[36]$.

If $t=t_{n}$, it then follows from (23) and using the inequality $x^{\top} y+y^{\top} x \leq \varepsilon x^{\top} x+\varepsilon^{-1} y^{\top} y$ that we can obtain

$$
\begin{aligned}
& \left(1+2 \lambda_{c}\left(L^{(n) w}\right)+\lambda_{d}\left(L^{(n) \top} L^{(n)}\right)\right) V\left(t_{n}\right) \\
& \leq e^{\top}\left(t_{n}\right)\left(I+L^{(n) \top}\right)\left(I+L^{(n)}\right) e\left(t_{n}\right) \\
& \leq\left\{e^{\top}\left(t_{n}^{-}\right)-\left[\sum_{s=n-\xi_{n}+1}^{n} L^{(n)} L^{(s)} e\left(t_{s}-\tau\left(t_{s}\right)\right)\right.\right. \\
& +L^{(n)} \sum_{j=1}^{\xi_{n}} \int_{t_{n-j}}^{t_{n-j+1}^{-}} B^{(n-j)} e(t) d t \\
& \left.\left.-L^{(n)} \int_{t_{n-\xi_{n}}}^{t_{n}-\tau\left(t_{n}\right)} B^{\left(n-\xi_{n}\right)} e(t) d t\right]^{\top}\right\} \\
& \times\left\{e ( t _ { n } ^ { - } ) \left[\sum_{s=n-\xi_{n}+1}^{n} L^{(n)} L^{(s)} e\left(t_{s}-\tau\left(t_{s}\right)\right)\right.\right. \\
& +L^{(n)} \sum_{j=1}^{\xi_{n}} \int_{t_{n-j}}^{t_{n-j+1}^{-}} B^{(n-j)} e(t) d t \\
& \left.\left.-L^{(n)} \int_{t_{n-\xi_{n}}}^{t_{n}-\tau\left(t_{n}\right)} B^{\left(n-\xi_{n}\right)} e(t) d t\right]\right\}
\end{aligned}
$$

$$
\begin{aligned}
& \leq V\left(t_{n}^{-}\right)+\xi_{n}\left\|L^{(n)}\right\| \max _{n-\xi_{n}+1 \leq s \leq n}\left\|L^{(s)}\right\| \\
& \times\left\{e^{\top}\left(t_{n}^{-}\right)\left[e\left(t_{n}\right)\right]_{2 \tau^{-}}+e\left(t_{n}^{-}\right)\left[e\left(t_{n}\right)\right]_{2 \tau^{-}}^{\top}\right\} \\
& +\left(\xi_{n}+1\right) \Delta\left\|L^{(n)}\right\| \max _{n-\xi_{n} \leq l \leq n-1}\left\|B^{(l)}\right\| \\
& \times\left\{e^{\top}\left(t_{n}^{-}\right)\left[e\left(t_{n}\right)\right]_{2 \tau^{-}}+e\left(t_{n}^{-}\right)\left[e\left(t_{n}\right)\right]_{2 \tau^{-}}^{\top}\right\} \\
& +\xi_{n}^{2}\left\|L^{(n)}\right\|_{n-\xi_{n}+1 \leq s \leq n}^{2}\left\|L^{(s)}\right\|^{2}\left(\left[e\left(t_{n}\right)\right]_{2 \tau^{-}}^{\top}\left[e\left(t_{n}\right)\right]_{2 \tau^{-}}\right) \\
& +\xi_{n}\left(\xi_{n}+1\right) \Delta\left\|L^{(n)}\right\|_{n-\xi_{n}+1 \leq s \leq n}^{2}\left\|L^{(s)}\right\| \\
& \times \max _{n-\xi_{n} \leq l \leq n-1}\left\|B^{(l)}\right\|\left(\left[e\left(t_{n}\right)\right]_{2 \tau^{-}}^{\top}\left[e\left(t_{n}\right)\right]_{2 \tau^{-}}\right) \\
& +\left(\xi_{n}+1\right)^{2} \Delta^{2}\left\|L^{(n)}\right\|^{2} \\
& \times \max _{n-\xi_{n} \leq l \leq n-1}\left\|B^{(l)}\right\|^{2}\left(\left[e\left(t_{n}\right)\right]_{2 \tau^{-}}^{\top}\left[e\left(t_{n}\right)\right]_{2 \tau^{-}}\right) \\
& \leq\left\{1+\varepsilon \xi_{n}\left\|L^{(n)}\right\| \max _{n-\xi_{n}+1 \leq s \leq n}\left\|L^{(s)}\right\|\right. \\
& \left.+\varepsilon\left(\xi_{n}+1\right) \Delta\left\|L^{(n)}\right\|_{n-\xi_{n} \leq l \leq n-1}\left\|B^{(l)}\right\|\right\} V\left(t_{n}^{-}\right) \\
& +\left\{\varepsilon^{-1} \xi_{n}\left\|L^{(n)}\right\| \max _{n-\xi_{n}+1 \leq s \leq n}\left\|L^{(s)}\right\|\right. \\
& +\varepsilon^{-1}\left(\xi_{n}+1\right) \Delta\left\|L^{(n)}\right\| \max _{n-\xi_{n} \leq l \leq n-1}\left\|B^{(l)}\right\| \\
& +\xi_{n}^{2}\left\|L^{(n)}\right\|_{n-\xi_{n}+1 \leq s \leq n}^{2}\left\|L^{(s)}\right\|^{2} \\
& +\left(\xi_{n}+1\right)^{2} \Delta^{2}\left\|L^{(n)}\right\|^{2} \max _{n-\xi_{n} \leq l \leq n-1}\left\|B^{(l)}\right\|^{2} \\
& +\xi_{n}\left(\xi_{n}+1\right) \Delta\left\|L^{(n)}\right\|^{2} \\
& \left.\times \max _{n-\xi_{n}+1 \leq s \leq n}\left\|L^{(s)}\right\| \max _{n-\xi_{n} \leq l \leq n-1}\left\|B^{(l)}\right\|\right\}\left[V\left(t_{n}\right)\right]_{2 \tau^{-}} .
\end{aligned}
$$

Then from the above proof, we can obtain

$$
\begin{gathered}
D^{+} V(t) \leq 0, \quad t \neq t_{n}, \quad t \geq 0, n \in \mathbb{N}, \\
V\left(t_{n}^{+}\right) \leq p_{n} V\left(t_{n}^{-}\right)+q_{n}\left[V\left(t_{n}\right)\right]_{2 \tau^{-}}, \quad t=t_{n},
\end{gathered}
$$

where $p_{n}, q_{n}$ are defined in Theorem 3; then according to Lemma 1 in [33], consensus of the system (2) is achieved globally exponentially. That completes the proof of Theorem 3.

Remark 4. The result of Theorem 2 (Theorem 3) indicates that the topology structure is an important factor in achieving consensus. The communication delay $\tau$ and the interval $t_{n}-$ $t_{n-1}$ of information exchanging also affect the consensus of 


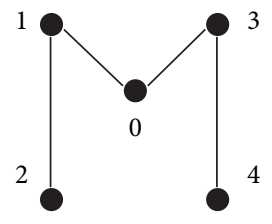

(a) $\widetilde{G}_{1}$ and $G_{1}$

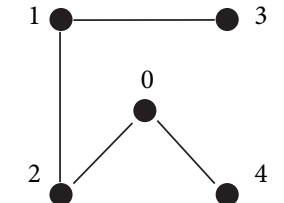

(b) $\widetilde{G}_{2}$ and $G_{2}$

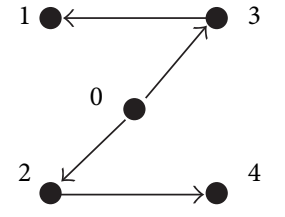

(c) $\widetilde{G}_{3}$ and $G_{3}$

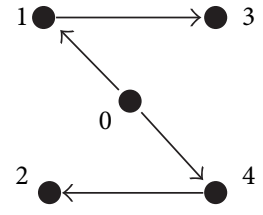

(d) $\widetilde{G}_{4}$ and $G_{4}$

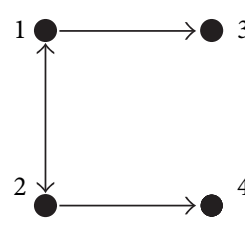

(e) $G_{5}$

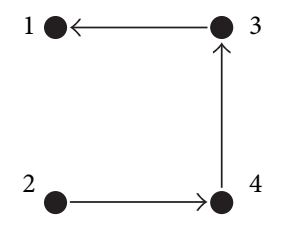

(f) $G_{6}$

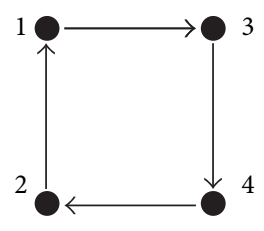

(g) $G_{7}$

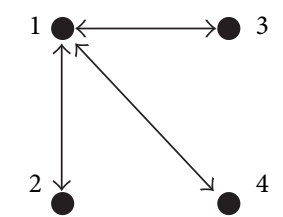

(h) $G_{8}$

Figure 1: Topologies (a)-(d) are studied by our model, whereas topologies (e)-(h) are studied by [9, 10, 22, 23].

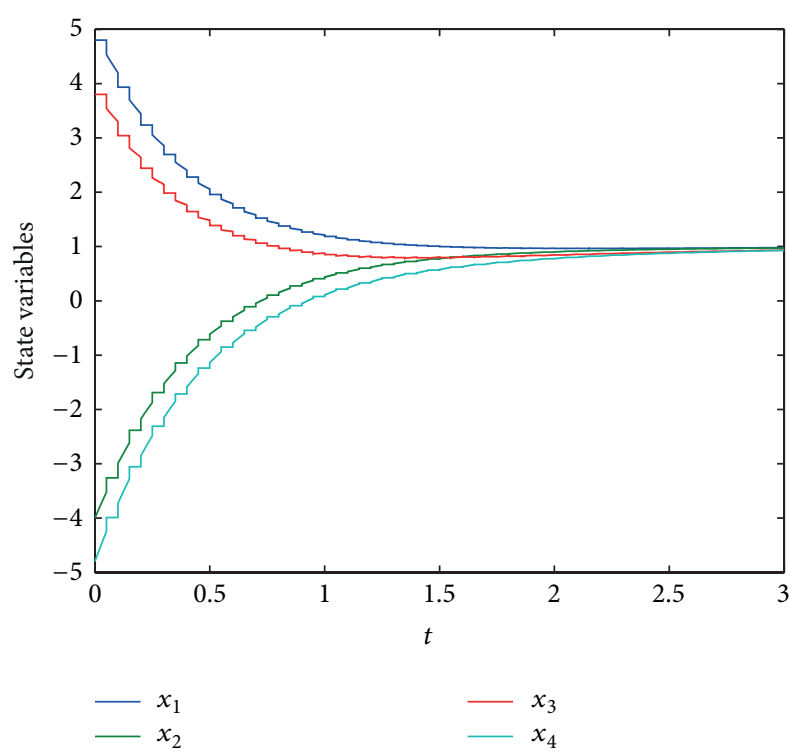

(a)

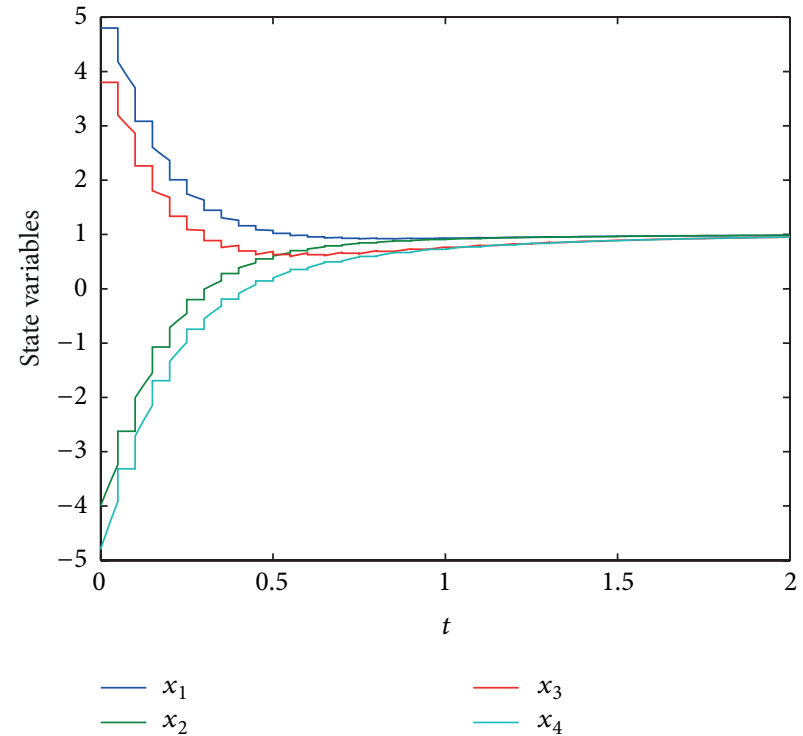

(b)

Figure 2: The trajectories of agents in the system (2) having different weighting factors over stochastic switching topologies $\left\{\widetilde{G}_{1}, \widetilde{G}_{2}\right\}$. (a) $\tau=0.1, d=0.03, \alpha=2$; (b) $\tau=0.1, d=0.07, \alpha=3.3$.

the system (2). Therefore, for a delayed dynamical network to achieve consensus, we need to adjust the topology structure, the time delay, and the interval of information exchanging so that the condition of Theorem 2 (Theorem 3 ) is satisfied. In the next section, we will explain this point through numerical simulations.

Remark 5. In this paper, only sufficient condition is derived; that is, the system (2) can achieve consensus although the condition (7) or condition (19) may fail. Theorem 2 is only used for undirected network topology, where both undirected and directed network topology can be dealt with by Theorem 3, yet we will show that Theorem 2 is more suitable than Theorem 3 to handle the undirected graphs in the next section.

Remark 6. In [9], Olfati-Saber and Murray defined the communication or sensing cost of the topology $G(V, E, A)$.
In this paper, we give a similar definition of the average communication cost of switching topology $G^{(n)}(V, E, A)$ in a multiagent system as follows [9]:

$$
C=\min \frac{1}{\zeta} \sum_{n=1}^{\zeta} \sum_{i, j=1}^{m} a_{i j}^{(n)}
$$

s.t. system achieve consensus at a given time,

where $a_{i j}^{(n)}$ is an element of weighted adjacency matrix $A^{(n)}$ of a network topology $G^{(n)}$ at $t_{n}$ and $\zeta$ denotes the total number of all interconnection graphs. Here we only give some discussions about cost through simulations and take the directed network with switching topology, for example, in the next section. We will show some advantages such as time-saving and energy-saving of our proposed algorithm. 


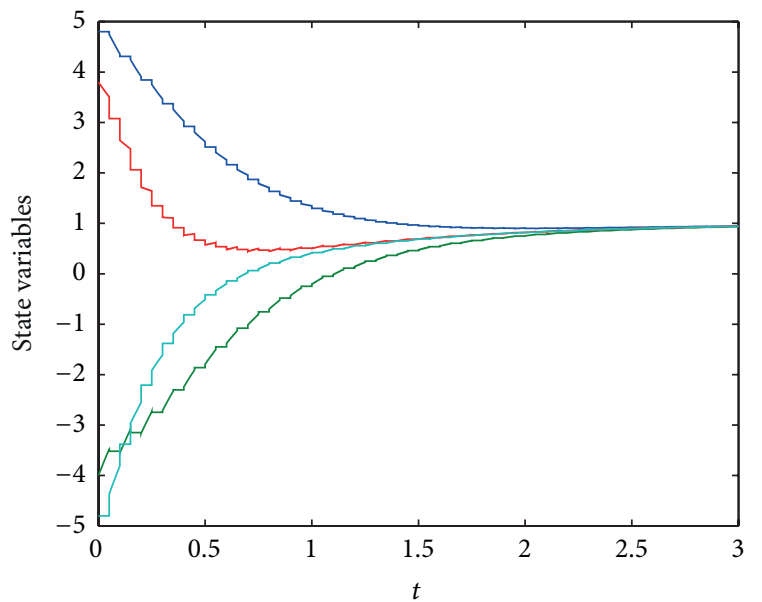

(a)

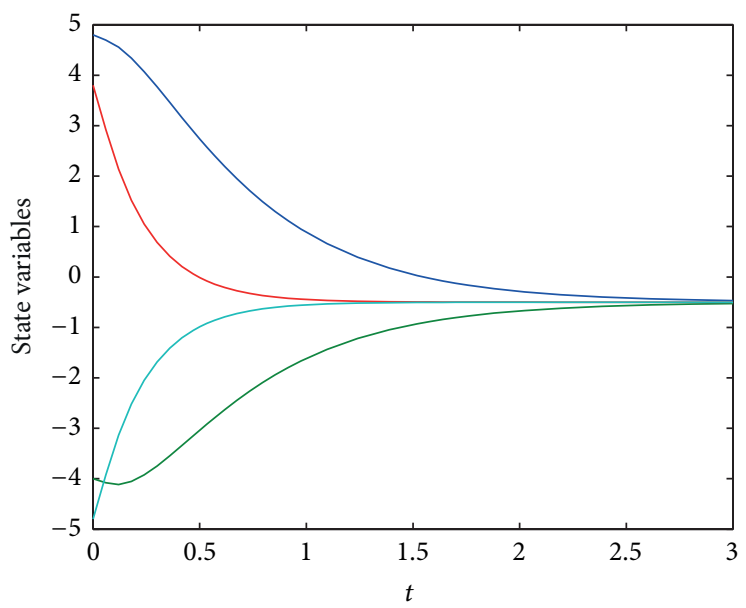

(c)

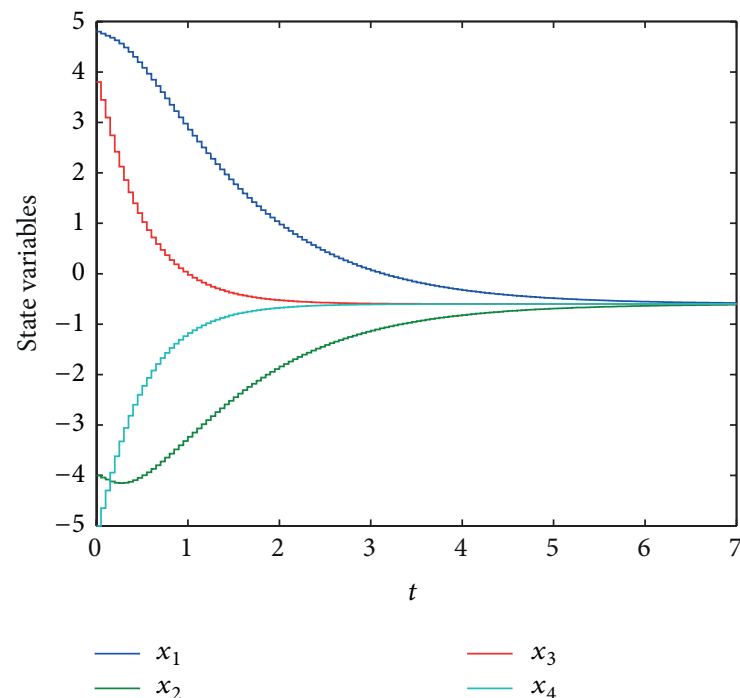

(e)

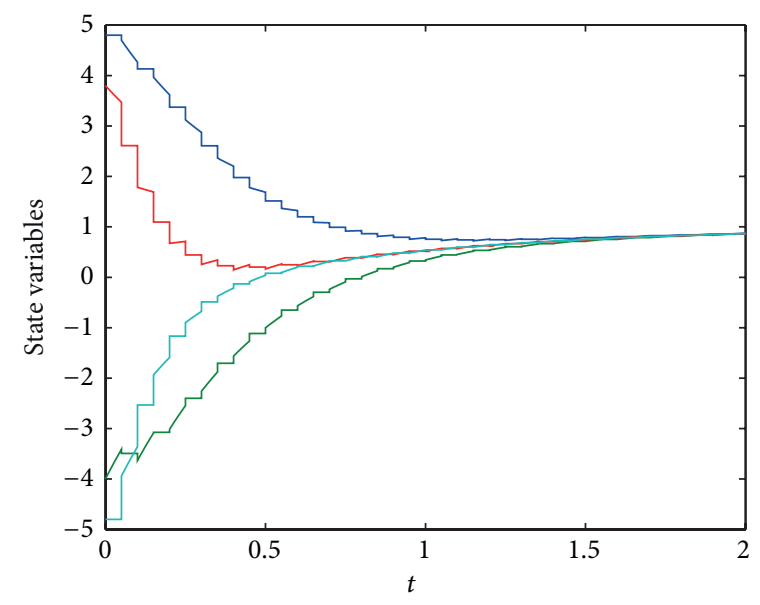

(b)

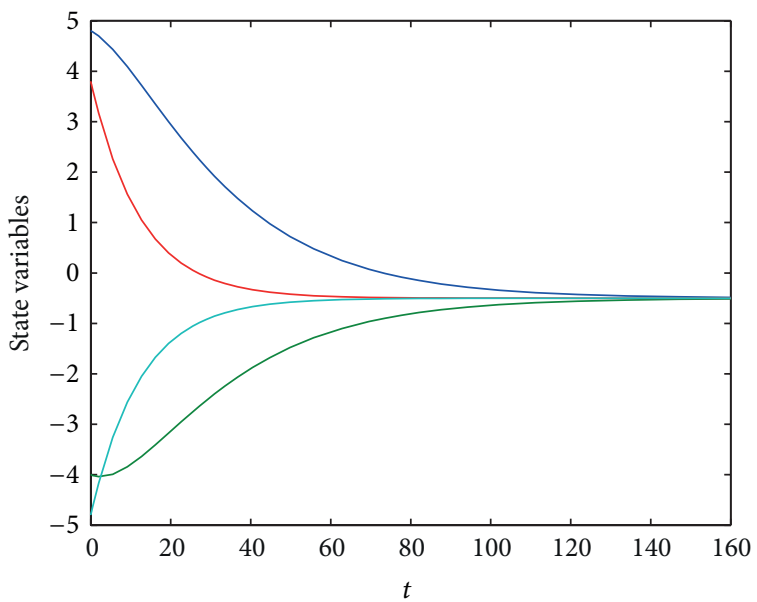

(d)

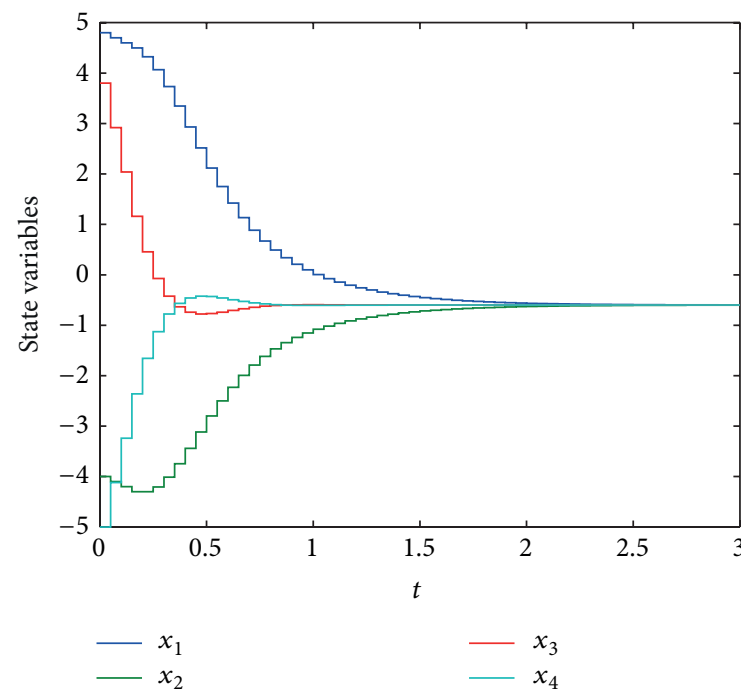

(f)

FIGURE 3: (a)-(b) are figures of system (2), whereas (c)-(d) are figures of system (1) studied by [9, 10], (e)-(f) are figures of the models studied by $[22,23]$. (a) $\tau=0.07, d=0.04, \alpha=2.2$; (b) $\tau=0.07, d=0.1, \alpha=2.5$; (c) $\tau=0.07, d=1.7$; (d) $\tau=0.07, d=0.04$; (e) $\tau=0.07, d=0.04$; (f) $\tau=0.07, d=1.2$. 
Remark 7. Wu et al. [22] and Guan et al. [23] investigated impulsive consensus problems without a leader for multiagent systems. As we know, in some cases, the coupled network is required to reach some prescribed consensus value, for example, $x_{0}$. Unlike $[22,23]$, we can drive the system to achieve the required consensus value by controlling a leader. Moreover, our model shows a faster convergence and lesser cost than the models of $\mathrm{Wu}$ and Guan via simulation in the next section.

\section{Simulations}

In this section, we provide simulations to illustrate the effectiveness and advantages of the proposed algorithms. Obviously, from Figure 1, we can see that graphs Figures 1(a)-1(d) formed by followers do not have a spanning tree. But for all network communication topologies studied by our model, node 0 is globally reachable. We suppose that the leader's position described by (3) is $x_{0}=1$.

4.1. Undirected Networks over Switching Topology. In this simulation, the interaction topology switches randomly every $0.1 \mathrm{~s}$ among $\left\{\widetilde{G}_{1}, \widetilde{G}_{2}\right\}$ as shown in Figure 1. We suppose that $t_{n}-t_{n-1} \equiv \Delta t=0.05, \tau(t)=0.07+0.03 \cos (t)$ with the maximum time delay $\tau=0.1$. And we stochastically choose initial values in $[-5,5]$. Choosing the parameters $d=0.03, \alpha=2, \lambda=0.5$, to satisfy condition (7) of Theorem 2, Figure 2(a) indicates that the system (2) achieves consensus globally exponentially. By Theorem 3 , use the same parameters $d=0.03, \alpha=2$, but there does not exist $\lambda>0$ to satisfy condition (19). Then we need to make the maximum time-delay $\tau$ smaller, for example, $\tau=0.04<\Delta t$, to satisfy condition (19). So we say that Theorem 2 is more suitable for undirected networks, as discussed in Remark 5. Figure 2(b) implies that the condition of Theorem 2 is a sufficient condition but not necessary (the parameters $d=$ $0.07, \alpha=3.3$ dissatisfy the condition (7)).

4.2. Directed Networks over Switching Topology. In this simulation, the interaction topology switches randomly every $0.1 \mathrm{~s}$ among $\left\{\widetilde{G}_{3}, \widetilde{G}_{4}\right\}$ as shown in Figure 1 . We suppose that $t_{n}-$ $t_{n-1} \equiv \Delta t=0.05, \tau(t)=0.06+0.01 \sin (t)$, with the maximum time delay $\tau=0.07$. And we stochastically choose initial values in $[-5,5]$. It is easy to see that the condition (19) of Theorem 3 is satisfied if $d=0.04, \alpha=2.2, \lambda=0.5, \varepsilon=$ 1. Figure 3(a) shows that the system (2) achieves consensus globally exponentially. Figure 3(b) illustrates that we only derive a sufficient condition, as discussed in Remark 5 (the parameters $d=0.1, \alpha=2.5$ dissatisfied (19) of Theorem 3).

\subsection{Performance and Cost of Our Model via Simulation.} In this section, we address advantages of our model via simulation as discussed in Remarks 6 and 7. Suppose that the system achieves consensus at exactly three seconds according to (27); then system (2) have the communication cost $C=$ 4.48 , where the system (1) studied by $[9,10]$ requires more cost $(C=6.8)$ to achieve consensus as shown in Figure 3(c).
Then we say our proposed protocol is energy-saving and costsaving as discussed in Remark 6. Figure 3(d) suggests that the system (1) needs more time ( $t=160 \mathrm{~s})$ to achieve consensus if the parameters chosen are the same $(d=0.04, \tau(t)=0.06+$ $0.01 \sin (t))$ as those in Figure 3(a). So we say our proposed protocol is time-saving as discussed in Remark 6. Compared with $[22,23]$, we introduce a leader in a delayed multiagent system, and we can drive the system to any desired consensus value by controlling a leader. Figures 3(e) and 3(f) show that the model studied by works of $[22,23]$ needs more time $(t=$ $7 \mathrm{~s}$ when parameters chosen are the same as Figure 3(a)) and more cost ( $C=5$ according to (27)) than system (2).

\section{Conclusion}

In this paper, we study leader-following consensus problem of delayed multiagent systems with impulsive effects over a general network topology and propose a protocol based on sample data information. Some sufficient conditions are obtained to guarantee that the followers converge to the leader globally exponentially. We show that under this impulsive protocol, the delayed networks can achieve consensus even the followers exchange information with each other only at some discrete moments. Some simulations are given to verify the effectiveness of the theoretical results. The timesaving and energy-saving of the protocol are also shown via simulations.

\section{Conflict of Interests}

The authors declare that there is no conflict of interests regarding the publication of this paper. This paper is new. Neither the entire paper nor any part of its content has been published or has been accepted elsewhere. It is not being submitted to any other journal. All authors have seen the manuscript and approved to submit to your journal.

\section{Acknowledgments}

The authors are grateful to the Associate Editor and the anonymous reviewers for their valuable comments and suggestions that helped improve the presentation of the paper. This work was partially supported by the National Science Foundation of China (Grant no. 61364003) and the Science and Technology Foundation of Guizhou Province (no. 20122316).

\section{References}

[1] C. W. Reynolds, "Flocks, herds, and schools: a distributed behavior model," Computer Graphics, vol. 21, no. 4, pp. 25-34, 1987.

[2] R. Olfati-Saber, "Flocking for multi-agent dynamic systems: algorithms and theory," IEEE Transactions on Automatic Control, vol. 51, no. 3, pp. 401-420, 2006.

[3] D. P. Spanos and R. M. Murray, "Distributed sensor fusion using dynamic consensus," in Proceedings of the 16th IFAC World Congress, Venue, Czech Republic, 2005, We-E04-TP/3. 
[4] P. K. C. Wang and F. Y. Hadaegh, "Coordination and control of multiple microspacecraft moving in formation," Journal of the Astronautical Sciences, vol. 44, no. 3, pp. 315-355, 1996.

[5] T. Balch and R. C. Arkin, "Behavior-based formation control for multirobot teams," IEEE Transactions on Robotics and Automation, vol. 14, no. 6, pp. 926-939, 1998.

[6] T. Vicsek, A. Czirk, E. Ben-Jacob, I. Cohen, and O. Shochet, "Novel type of phase transition in a system of self-driven particles," Physical Review Letters, vol. 75, no. 6, pp. 1226-1229, 1995.

[7] A. Jadbabaie, J. Lin, and A. S. Morse, "Coordination of groups of mobile autonomous agents using nearest neighbor rules," IEEE Transactions on Automatic Control, vol. 48, no. 6, pp. 988-1001, 2003.

[8] A. V. Savkin, "Coordinated collective motion of groups of autonomous mobile robots: analysis of Vicsek's model," IEEE Transactions on Automatic Control, vol. 49, no. 6, pp. 981-983, 2004.

[9] R. Olfati-Saber and R. M. Murray, "Consensus problems in networks of agents with switching topology and time-delays," IEEE Transactions on Automatic Control, vol. 49, no. 9, pp. 15201533, 2004.

[10] W. Ren and R. W. Beard, "Consensus seeking in multiagent systems under dynamically changing interaction topologies," IEEE Transactions on Automatic Control, vol. 50, no. 5, pp. 655661, 2005.

[11] C.-Y. Kao and A. Rantzer, "Stability analysis of systems with uncertain time-varying delays," Automatica, vol. 43, no. 6, pp. 959-970, 2007.

[12] D. J. Lee and M. W. Spong, "Agreement with non-uniform information delays," in Proceedings of the American Control Conference, pp. 756-761, Minneapolis, Minn, USA, June 2006.

[13] J. Q. Lu, D. W. C. Ho, and J. Kurths, "Consensus over directed static networks with arbitrary finite communication delays," Physical Review E, vol. 80, no. 6, Article ID 066121, 2009.

[14] X. Liu, W. Lu, and T. Chen, "Consensus of multi-agent systems with unbounded time-varying delays," IEEE Transactions on Automatic Control, vol. 55, no. 10, pp. 2396-2401, 2010.

[15] K. Li, "Impulsive effect on global exponential stability of BAM fuzzy cellular neural networks with time-varying delays," International Journal of Systems Science, vol. 41, no. 2, pp. 131142, 2010.

[16] T. Yang and L. O. Chua, "Impulsive stabilization for control and synchronization of chaotic systems: theory and application to secure communication," IEEE Transactions on Circuits and Systems I, vol. 44, no. 10, pp. 976-988, 1997.

[17] J. Zhou, H. Zhang, L. Xiang, and Q. Wu, "Synchronization of coupled harmonic oscillators with local instantaneous interaction," Automatica, vol. 48, no. 8, pp. 1715-1721, 2012.

[18] O. C. Imer, S. Yüksel, and T. Başar, "Optimal control of LTI systems over unreliable communication links," Automatica, vol. 42, no. 9, pp. 1429-1439, 2006.

[19] D. W. C. Ho and J. Sun, "Stability of takagi-sugeno fuzzy delay systems with impulse," IEEE Transactions on Fuzzy Systems, vol. 15, no. 5, pp. 784-790, 2007.

[20] P. Li, J. Cao, and Z. Wang, "Robust impulsive synchronization of coupled delayed neural networks with uncertainties," Physica A, vol. 373, pp. 261-272, 2007.

[21] L. Zhang and H. Jiang, "Impulsive generalized synchronization for a class of nonlinear discrete chaotic systems," Communications in Nonlinear Science and Numerical Simulation, vol. 16, no. 4, pp. 2027-2032, 2011.
[22] Q. Wu, J. Zhou, H. Zhang, and X. Lan, "Distributed $\delta$ consensus in directed delayed networks of multi-agent systems," International Journal of Systems Science, vol. 44, no. 5, pp. 916925, 2013.

[23] Z.-H. Guan, Y. Wu, and G. Feng, "Consensus analysis based on impulsive systems in multiagent networks," IEEE Transactions on Circuits and Systems I, vol. 59, no. 1, pp. 170-178, 2012.

[24] H. Zhang and J. Zhou, "Distributed impulsive consensus for second-order multi-agent systems with input delays," IET Control Theory \& Applications, vol. 7, no. 16, pp. 1978-1983, 2013.

[25] D. Hummel, "Formation flight as an energy-saving mechanism," Israel Journal of Zoology, vol. 41, no. 3, pp. 261-278, 1995.

[26] M. Andersson and J. Wallander, "Kin selection and reciprocity in flight formation?" Behavioral Ecology, vol. 15, no. 1, pp. 158$162,2004$.

[27] Y. Hong, J. Hu, and L. Gao, "Tracking control for multiagent consensus with an active leader and variable topology," Automatica, vol. 42, no. 7, pp. 1177-1182, 2006.

[28] J. Hu and Y. Hong, "Leader-following coordination of multiagent systems with coupling time delays," Physica A, vol. 374, no. 2, pp. 853-863, 2007.

[29] Q. Song, J. Cao, and W. Yu, "Second-order leader-following consensus of nonlinear multi-agent systems via pinning control," Systems and Control Letters, vol. 59, no. 9, pp. 553-562, 2010.

[30] Z. Wang and J. Cao, "Quasi-consensus of second-order leaderfollowing multi-agent systems," IET Control Theory and Applications, vol. 6, no. 4, pp. 545-551, 2012.

[31] S. Yang, J. Cao, and J. Lu, "A new protocol for finite-time consensus of detail-balanced multiagent networks," Chaos, vol. 22, no. 4, Article ID 043134, 2012.

[32] Q. Song, F. Liu, J. Cao, and W. Yu, "M-matrix strategies for pinning-controlled leader- following consensus in multiagent systems with nonlinear dynamics," IEEE Transactions on Cybernetics, vol. 43, no. 6, pp. 1688-1697, 2013.

[33] Q. Wu, J. Zhou, X. Lan, and S. Cheng, "Impulsive control and synchronization for a class of chaotic delayed systems," in Proceedings of the 29th Chinese Control Conference (CCC '10), pp. 514-519, Beijing, China, July 2010.

[34] W. Yu, G. Chen, M. Cao, and J. Kurths, "Second-Order consensus for multiagent systems with directed topologies and nonlinear dynamics," IEEE Transactions on Systems, Man, and Cybernetics B, vol. 40, no. 3, pp. 881-891, 2010.

[35] Q. Zhang, S. Chen, and C. Yu, "Impulsive consensus problem of second-order multi-agent systems with switching topologies," Communications in Nonlinear Science and Numerical Simulation, vol. 17, no. 1, pp. 9-16, 2012.

[36] W. Ren and Y. Cao, Distributed Coordination of Multi-Agent Networks: Emergent Problems, Models, and Issues, Springer, London, UK, 2011. 


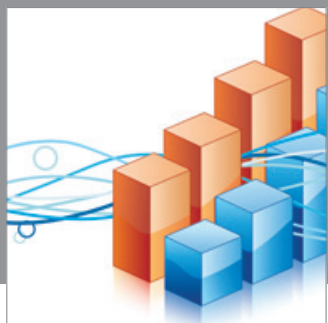

Advances in

Operations Research

mansans

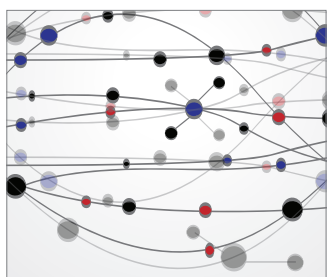

The Scientific World Journal
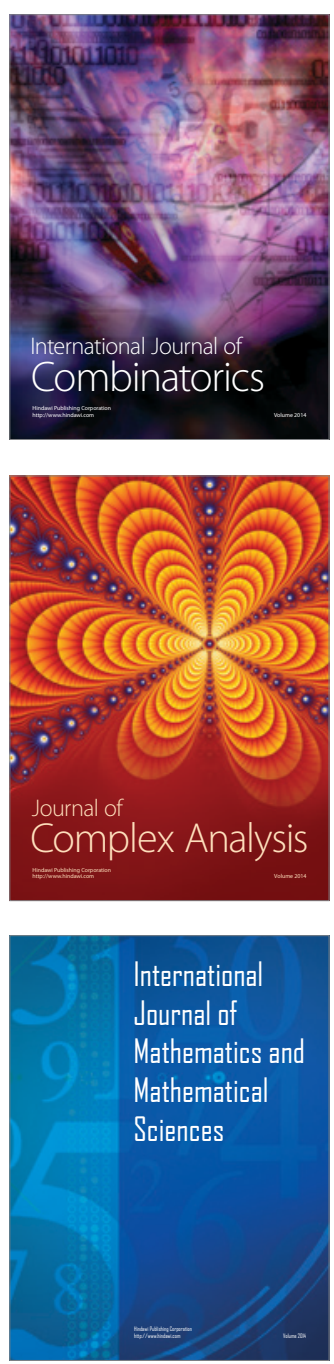
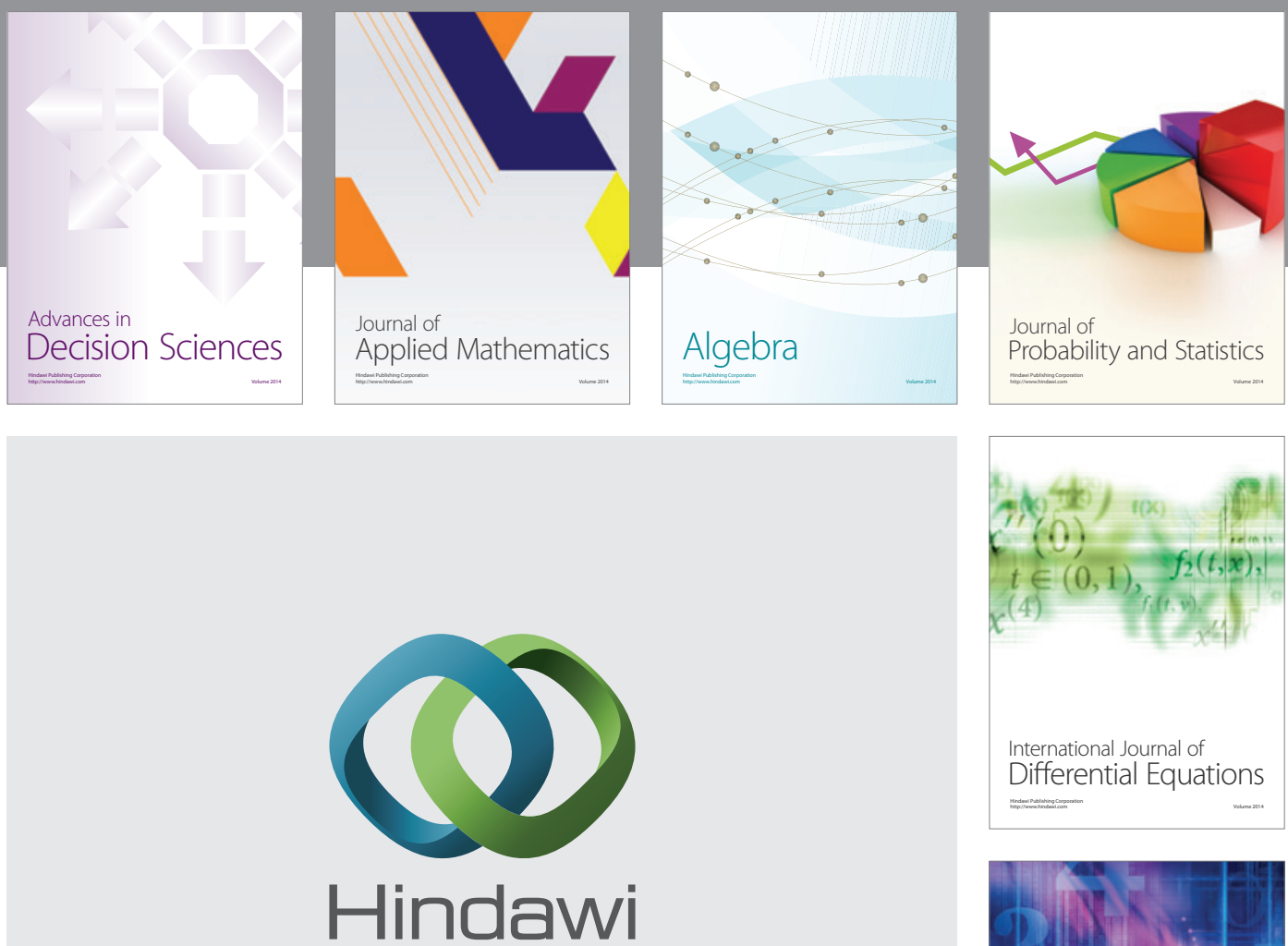

Submit your manuscripts at http://www.hindawi.com
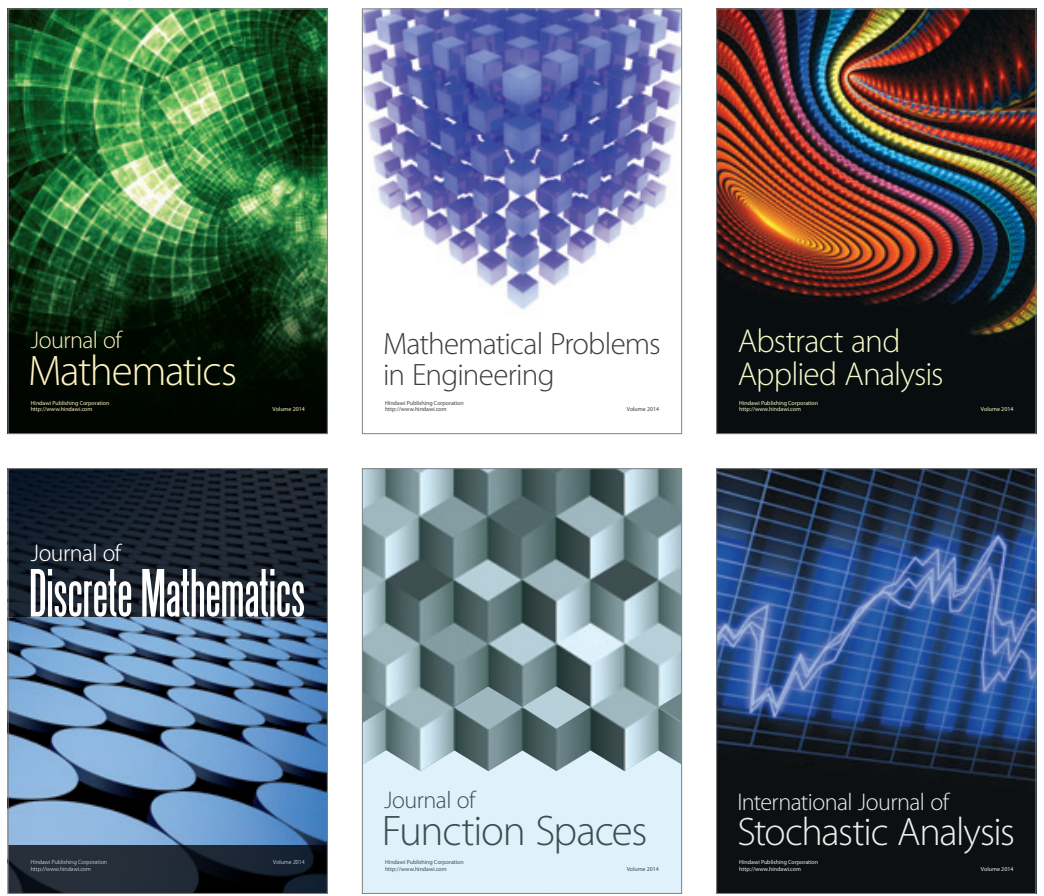

Journal of

Function Spaces

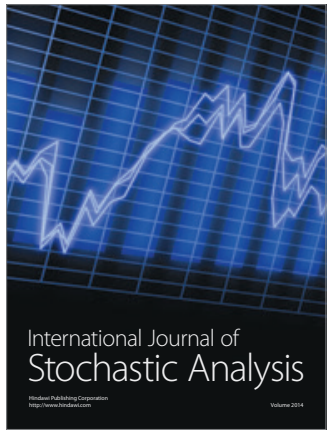

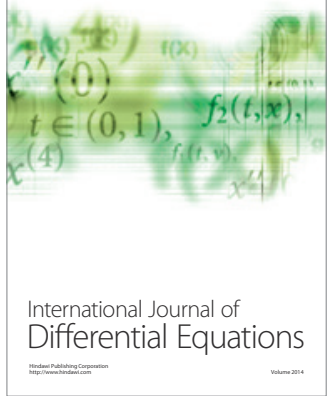
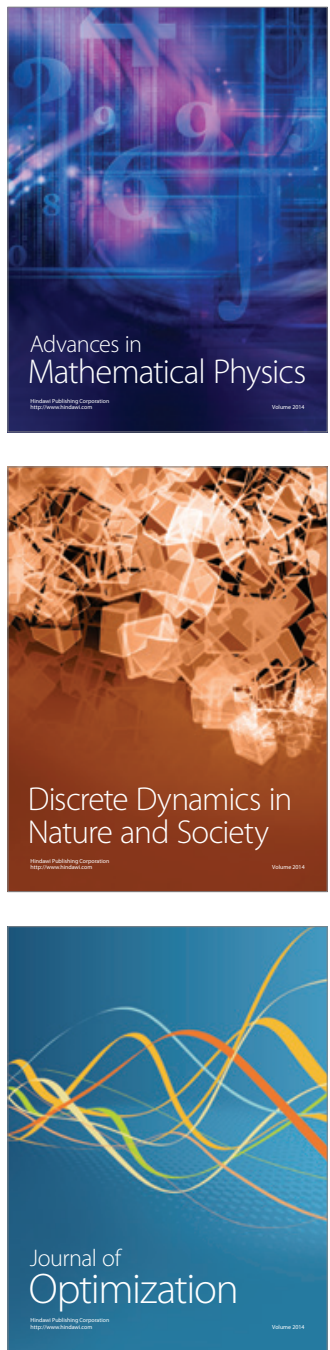